\title{
Revising Elastic-Plastic Contact Models of Fractal Surfaces
}

\author{
Cuicui $\mathrm{Ji}^{1, \mathrm{a}}$, Wei Jiang ${ }^{2, \mathrm{~b}}$ \\ ${ }^{1}$ School of Mechanical and Electrical Engineering, Hohai University, \\ Changzhou, Jiangsu 213022, PR China \\ ${ }^{2}$ School of Mechanical and Automobile Engineering, Changzhou Institute of Technology, \\ Changzhou, Jiangsu 213002, PR China \\ a20121856@hhu.edu.cn, bbrucejw@126.com
}

\begin{abstract}
Keywords: contact; elastic-plastic; fractal surface; asperity deformation
Abstract. This paper conducts revisions of the existing elastic-plastic contact models for a single asperity with the fractal surface topographies generated from $\mathrm{W}-\mathrm{M}$ functions. The expressions of the critical parameters and the contact load-area relationship are deduced in the terms of fractal parameters, asperity's base diameter and material properties. Numerical analysis suggest that the contact models' critical parameters derived strictly in a convincing and easier way in this study are scale-dependent on the asperity's base size and determined jointly by fractal parameters, asperity's base diameter and material properties. The transformation condition for an asperity transfers from elastic to plastic contact and the contact load-area relationship are consistent with those in classical contact mechanics but contrary to the existing fractal contact models.
\end{abstract}

\section{Introduction}

Due to the existence of surface roughness, two surfaces' real contact only occurs at the asperities occupying a very small proportion of the nominal contact area. Therefore, the contact mechanics of rough surfaces is very crucial for tribology study such as friction, adhesion, wear etc. During the last forty years, many researchers have dedicated to working out the problem of the contact between rough surfaces. Therefore, a large number of contact models based on statistical or fractal topography parameters have been developed [1-3]. Majumdar and Bhushan [3] developed the first fractal contact model, i.e. M-B model. For a signal elastically deformed asperity contact, there are two disagreements between the M-B model and the classical contact models, such as Hertz contact theory. Firstly, the relationship between the real contact area $a$, and the load $F$ obtained from the M-B model is of the form: $a \propto F^{(3-D) / 2}(1<D<2)$, while that from the Hertz model is $a \propto F^{3 / 2}$. Secondly, in the M-B model, when the contact area $a<a_{\mathrm{c}}\left(a_{\mathrm{c}}\right.$ is the critical contact area,), the contact mode is plastic, and transfers to be elastic when $a>a_{c}$; whereas, the reversal is true for the situation in classical contact models. Soon after, Wang and Komvopoulos [4-6] modified the M-B model. Yan and Komvopoulos [7] further modified the M-B model using a 3-dimensional (3-D) isotropic fractal surface also generated from Weierstrass and Mandelbrot (W-M) function [8]. Then these fractal contact models have been used in various fields of applied physics [9-12] without any doubts about the above-mentioned disagreements.

Until recently, Morag and Etsion [13] revised the M-B model of a single fractal asperity and found that the critical interference, the critical contact area and the critical load, are all scale-dependent. Their result also shows that the load and contact area increase a transition from elastic to plastic contact mode as takes place in the Hertz contact model and the relationship between the contact area and the load satisfies $a \propto F^{3 / 2}$. Morag and Etsion's study has provided an insight into the different outcomes in the M-B model and patched up the disagreements between the M-B model and Hertz model. However, there still exists unconvincing arguments which also can be further simplified in their study. Take Eq. 18 in Ref. [13] as an example, they replaced the asperity's critical height $\delta_{\mathrm{c}}$ with asperity's critical deformation $\omega_{\mathrm{c}}(0 \leq \omega \leq \delta)$ directly without any convincing explanation.

The main objective of this study is twofold. First, we intend to resolve the unconvincing aspects in Ref. [13] and then simplify its derivation process of the contact between a single asperity and a rigid 
flat plane. Second, we extend this revised contact mechanics analysis to the cases of the contact between 3-D fractal rough surfaces.

\section{Fractal Surface Characterization}

2-D Fractal Surface Two rough surfaces' contact is often simplified as an equivalent contact model consisting of a deformable rough surface with effective material properties and equivalent roughness in contact with a rigid plane [1]. In the M-B model, the rough surface is generated by 2-D W-M equation given in Eq. 1.

$$
z(x)=G^{(D-1)} \sum_{n=n_{l}}^{\infty} \gamma^{-(2-D) n} \cos \left(2 \pi \gamma^{n} x\right)
$$

where $D(1<\mathrm{D}<2)$ and $G$ are the fractal dimension and fractal roughness of the surface profile, respectively, both independent of the wavelength in the range where the surface exhibits fractal behaviors. $\gamma^{n}(\gamma>1)$ controls the density of frequency of the surface roughness and $\gamma=1.5$ is a typical value for most surfaces. $n$ is the spatial frequency index, which indicates the frequency level of the asperities, and the lowest level $n_{l}$ is related to the sampling length $L=1 / \gamma^{n l}$.

3-D Fractal Surface 3-D fractal rough surface can be generated from 3-D W-M function [7], as illustrated in Eq. 2.

$$
\begin{gathered}
z(x, y)=L(G / L)^{(D-2)}(\ln \gamma / M)^{1 / 2} \sum_{m=1}^{M} \sum_{n=n_{l}}^{n_{\text {max }}} \gamma^{(D-3) n} \times \\
\left\{\cos \phi_{m, n}-\cos \left[\begin{array}{l}
2 \pi \gamma^{n}\left(x^{2}+y^{2}\right)^{1 / 2} / L \times \\
\cos (\arctan (y / x)-\pi m / M)+\phi_{m, n}
\end{array}\right]\right\}
\end{gathered}
$$

where $D(2<D<3), G, \gamma, n$ and $L$ are the same as those in Eq. $1 . M$ is number of superposed ridges, and $\phi_{m, n}$, restricted in the range of $[0,2 \pi]$, is a random phase to prevent the coincidence of frequencies at point of the surface profile. Here, the lower limit of the frequency index $n$ is zero, while its upper limit is given by $n_{\max }=\operatorname{int}\left[\log \left(L / L_{s}\right) / \log \gamma\right]$, where $L_{s}$ is the cut-off length normally on the order of the material's inter-atomic distance.

\section{Contact Analysis}

Description of 2-D and 3-D Fractal Asperity The detailed description of the M-B model can be found in Ref. [3]. In this paper, only the most important and related parts of M-B model are listed. Fig. 1 displays a contact model between a rigid flat plane and a fractal asperity of which the geometry $z(x)$ is given in Eq. 3 .

$$
z(x)=G^{(D-1)} l^{(2-D)} \cos (\pi x / l),-l / 2<x<l / 2
$$

with the base diameter $l$ and frequency level $n=\log (1 / l) / \log \gamma$. The height of the asperity, $\delta$, is defined as in Eq. 4.

$$
\delta=z(0)=G^{(D-1)} l^{(2-D)}
$$

and Eq. 5 shows the asperity's radius of curvature $R$ as below

$$
R=1 /\left|\mathrm{d}^{2} z / \mathrm{d} x^{2}\right|_{x=0}=l^{D} /\left(\pi^{2} G^{(D-1)}\right)
$$

It is worth noting that, in the M-B model, the contact spot is assumed to be a circle with the base diameter $l$ while the contact area $a$ is set to be equal to $l^{2}$, i.e. $a \equiv l^{2}$. Then, Eqs. 4 and 5 can be written as in Eq. 6.

$$
\delta=G^{(D-1)} a^{(2-D) / 2} \text {, and } R=a^{D / 2} /\left(\pi^{2} G^{(D-1)}\right)
$$


According to Yan and Komvopoulos's study [7], for an isotropic 3-D fractal surface, an asperity's profile also with the base diameter $l$ can be given in Eq. 7 .

$$
\begin{aligned}
& z_{0}(x)=G^{(D-2)} \ln \gamma^{1 / 2} l^{(3-D)} \times \\
& {\left[\cos \phi_{1, n_{0}}-\cos \left(2 \pi x / l-\phi_{1, n_{0}}\right)\right]^{,-l / 2<x<l / 2}}
\end{aligned}
$$

the asperity's height $\delta$ and asperity's radius of curvature $R$ are shown in Eq. 8 .

$$
\delta=G^{(D-2)}(\ln \gamma)^{1 / 2} l^{(3-D)}, \text { and } R=1 /\left|\mathrm{d}^{2} z_{0} / \mathrm{d} x^{2}\right|_{x=0}=l^{(D-1)} /\left[4 \pi^{2} G^{(D-2)}(\ln \gamma)^{1 / 2}\right]
$$

It should be noticed that Eq. 9 is a little different from that in Ref. [7] in which $R$ is obtained using equation $l^{2} \approx 2 R \delta$. This will be described in detail later in this paper.

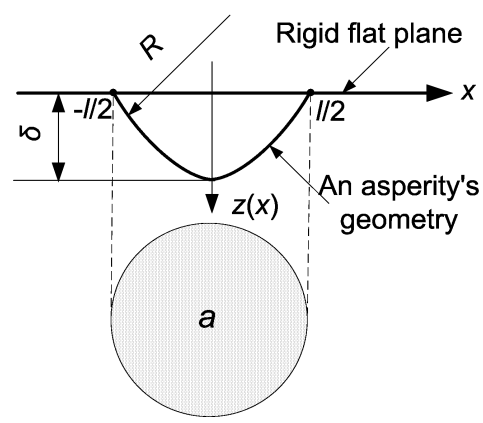

Figure 1: Schematic of a fractal asperity's contact with a rigid flat plan in M-B model.

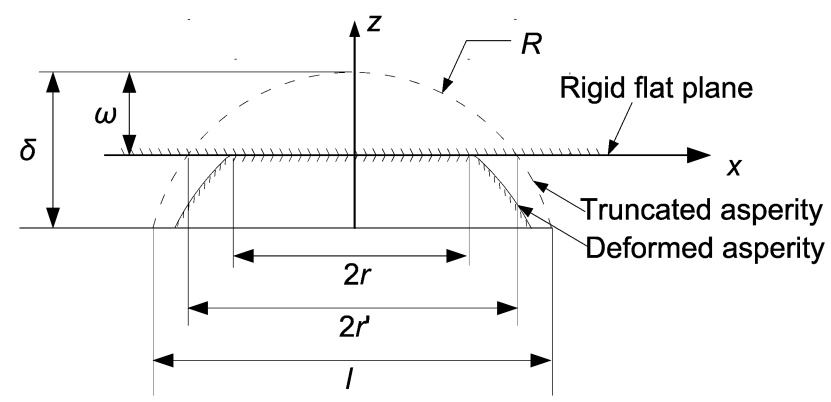

Figure 2: Schematic of a micro-contact established between a fractal asperity and a rigid flat plan of the revised contact model.

Revised Model of a 2-D Fractal Asperity Fig. 2 illustrates a truncated fractal asperity of scale $l$ with its profile $z(x)$, height $\delta$, and radius of curvature $R$ given by Eqs. 3 5, respectively. In the M-B model, the deformation $\omega$ is assumed to be identically equal to the asperity's height $\delta$, i.e. $\omega \equiv \delta$. In common with Ref. [13], the real deformation, $\omega$, ranging from zero to full deformation, i.e. $0 \leq \omega \leq \delta$, is introduced as an additional parameter independent of $\delta$. In addition, the deformation of any asperity in contact can be elastic or fully plastic in this study.

Eq. 9 shows the geometric relationships obtained from Fig. 2 as below

$$
a^{\prime}=\pi r^{\prime 2}=2 \pi r^{2}=2 a, \text { and } R^{2}=(l / 2)^{2}+(R-\delta)^{2}=r^{\prime 2}+(R-\omega)^{2}
$$

Due to the fact that $R$ is much larger than $\delta$, the part of $\delta^{2}$ can be omitted to obtain the following equations as demontrated in Eq. 10.

$$
r^{\prime 2} \approx 2 R \omega, l^{2} / 4 \approx 2 R \delta
$$

Then, the relationship between $\omega$ and $\delta$ is obtained as given in Eq. 11a.

$$
\omega=\delta \times\left(4 r^{\prime 2} / l^{2}\right)
$$

Substituting Eq. 4 into Eq. 11a results in Eq. 11b, which is shown below

$$
\omega=4 G^{(D-1)} l^{-D} r^{\prime 2}
$$

According to Ref. [1], the critical value of asperity interference (or deformation) $\omega_{c}$ at the inception of plastic flow can be written as in Eq. 12 .

$$
\omega_{\mathrm{c}}=b R(H / E)^{2}
$$

where $b$ is a constant independent of micro-contact size. The equivalent elastic modulus $E$ and hardness $H$ of the two contact surfaces are given as in Eq. 13 .

$$
1 / E=\left(1-v_{\mathrm{A}}^{2}\right) / E_{\mathrm{A}}+\left(1-v_{\mathrm{B}}^{2}\right) / E_{\mathrm{B}}, H=\min \left[H_{\mathrm{A}}, H_{\mathrm{B}}\right]
$$


where subscripts $\mathrm{A}$ and $\mathrm{B}$ refer to the two contacting surfaces, and $E, v$, and $H$ denote material's Young's elastic modulus, Poisson's ratio, and hardness. According to Eqs. 9, 11b and 12, the relationship between the critical contact area $a_{\mathrm{c}}$ and the critical interference $\omega_{\mathrm{c}}$ can be obtained in Eq. 14.

$$
\omega_{\mathrm{c}}=(8 / \pi) G^{(D-1)} l^{-D} a_{\mathrm{c}}
$$

According to Hertz contact theory, Eq. 15 shows the elastic and plastic load at signal micro-contact.

$$
\Delta F_{\mathrm{e}}=4 E r^{3} /(3 R), \text { and } \Delta F_{\mathrm{p}}=H a
$$

The normal force at a micro-contact is assumed to change continuously during the approach of the surfaces, thus, there exists $\Delta F_{\mathrm{e}}\left(a_{\mathrm{c}}\right)=\Delta F_{\mathrm{p}}\left(a_{\mathrm{c}}\right)$. Then, from Eqs. 5, 9, 15, $a_{\mathrm{c}}$ can be expressed in Eq. 16.

$$
a_{\mathrm{c}}=[9 /(16 \pi)](H / E)^{2}\left(l^{D} / G^{(D-1)}\right)^{2}
$$

Eq. 17 was obtained by substituting Eq. 16 into Eqs. 14 and 15.

$$
\begin{aligned}
\omega_{\mathrm{c}} & =(9 / 2)\left[l^{D} /\left(\pi^{2} G^{(D-1)}\right)\right](H / E)^{2} \\
& =(9 / 2) R(H / E)^{2}
\end{aligned}
$$

Simultaneously, the value of constant $b$ is $9 / 2$ by comparing Eqs. 13 and 16. It is easy to obtain the relationship between the contacting elastic load and the contacting area is shown in Eq. 18.

$$
\Delta F_{\mathrm{e}}=\left[4 E \pi^{1 / 2} G^{(D-1)} /\left(3 l^{D}\right)\right] a^{3 / 2}
$$

Revised Model of a 3-D Fractal Asperity The interference of 3-D fractal asperity can be easily obtained by introducing Eq. 8 into Eq. 11a and result in Eq. 19.

$$
\omega=4 G^{(D-2)}(\ln \gamma)^{1 / 2} l^{(1-D)} r^{\prime 2}
$$

Substituting Eqs. 8, 9, 15 into the equation $\Delta F_{\mathrm{e}}\left(a_{\mathrm{c}}\right)=\Delta F_{\mathrm{p}}\left(a_{\mathrm{c}}\right)$ is shown in Eq. 20.

$$
a_{\mathrm{c}}=[9 /(256 \pi \ln \gamma)](H / E)^{2}\left(l^{(D-1)} / G^{(D-2)}\right)^{2}
$$

and then $\omega_{\mathrm{c}}$ can be easily obtained as illustrated in Eq. 21 .

$$
\begin{aligned}
\omega_{\mathrm{c}} & =\left(9 l^{(D-1)}\right) /\left[32 \pi^{2} G^{(D-2)}(\ln \gamma)^{1 / 2}\right](H / E)^{2} \\
& =(9 / 8) R(H / E)^{2}
\end{aligned}
$$

with the value $b$ is $9 / 8$. In the same way, the relationship between the contacting elastic load and the contacting area of the 3-D fractal asperity is shown in Eq. 22.

$$
\Delta F_{\mathrm{e}}=\left[16 E \pi^{1 / 2} G^{(D-2)}(\ln \gamma)^{1 / 2} /\left(3 l^{(D-1)}\right)\right] a^{3 / 2}
$$

\section{Numerical Results and Discussion}

It is clear to see from the above equations that the critical parameters $\omega_{\mathrm{c}}$ and $a_{\mathrm{c}}$ are all scale-dependent in this study. This finding is in disagreement with that in Refs. [3] and [7] in which the critical areas are scale-dependent, whish is shown in Eq. 23.

$$
a_{\mathrm{c}}=G^{2} /[H /(2 E)]^{2 /(D-1)}, 1<D<2 \text { and } a_{\mathrm{c}}=\left\{2^{(11-2 D)} G^{(2 D-4)}(E / H)^{2} \ln \gamma / 9 \pi^{(4-D)}\right\}^{1 /(D-2)} / 2,2<D<3
$$

and Eq. 24 illustrates the further relationships between interference and contacting area.

$$
\delta_{\mathrm{c}} / \delta=\left(a / a_{\mathrm{c}}\right)^{(D-1)}, 1<D<2 ; \delta_{\mathrm{c}} / \delta=\left(a / a_{\mathrm{c}}\right)^{(D-2)}, 2<D<3
$$


The above four equations imply that an asperity with contact area $a>a_{\mathrm{c}}$ is elastically deformed since its deformation $\delta<\delta_{\mathrm{c}}$, whereas in contrary, an asperity with $a<a_{\mathrm{c}}$ is plastically deformed since its deformation $\delta>\delta_{\mathrm{c}}$. Namely, an increasing contact area leads to a transition from plastic to elastic contact mode, and the explanation given by Majumdar and Bhushan [3] is "due to the fact that smaller asperities have smaller radii of curvature and are therefore more likely to undergo plastic deformation". This result is contrary to that in classical contact mechanics, i.e. Hertz theory or G-W model [1]. The reason for this discrepancy is due to the assumption of $a \equiv l^{2}$ and $\omega \equiv \delta$ in M-B model. This study, with the assumption of the asperity's deformation $0 \leq \omega \leq \delta$ and curvature radius $R$ decided by its bases, is different from either the M-B model or the G-W model. The results of this study suggest that, the condition for an elastic contact transfers to a plastic contact is $\omega>\omega_{\mathrm{c}}$ corresponding to the condition $a>a_{\mathrm{c}}$, which is consistent with that in classical contact mechanics but contrary to that in the M-B model.

Table 1: Examples of contacting surfaces and their properties [11].

\begin{tabular}{|l|c|c|c|}
\hline \multirow{2}{*}{ Contact system } & \multicolumn{3}{|c|}{ Properties } \\
\cline { 2 - 4 } & Young's elastic modulus $E(\mathrm{Gpa})$ & Poisson's ratio $v$ & Hardness $H(\mathrm{Gpa})$ \\
\hline \multirow{2}{*}{$\mathrm{Al}_{2} \mathrm{O}_{3} / \mathrm{TiC}$} & 307 & 0.25 & 27.6 \\
\cline { 2 - 4 } & 450 & 0.18 & 23.5 \\
\hline \multirow{2}{*}{$\mathrm{Al}_{2} \mathrm{O}_{3} / \mathrm{CrN}$} & 307 & 0.25 & 27.6 \\
\hline \multirow{2}{*}{ AISI 1095/AISI 1020 } & 103 & 0.30 & 14.8 \\
\cline { 2 - 4 } & 200 & 0.29 & 6.08 \\
\hline
\end{tabular}

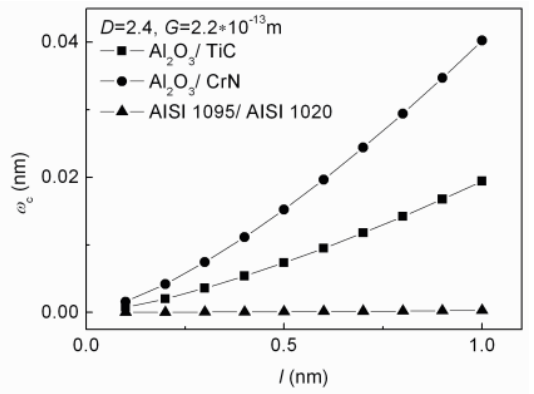

(a) $\omega_{c} v s . l$

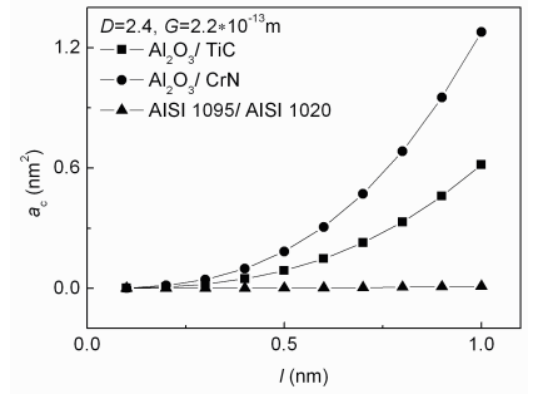

(b) $a_{c}$ vs. $l$

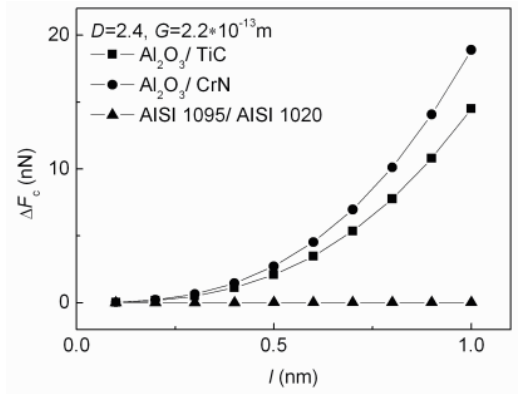

(c) $\Delta F_{e}\left(a_{c}\right)$ vs. $l$

Figure 3: Critical parameters versus the 3-D fractal asperity's base diameter 1 for the surface properties listed in Table 1.

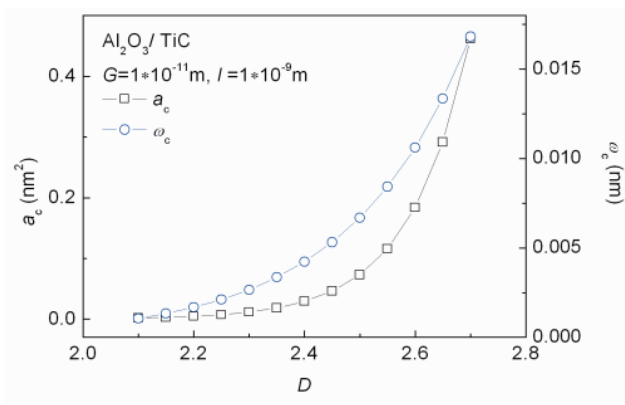

(a) $a_{c} \& \omega_{c}$ vs. $D$

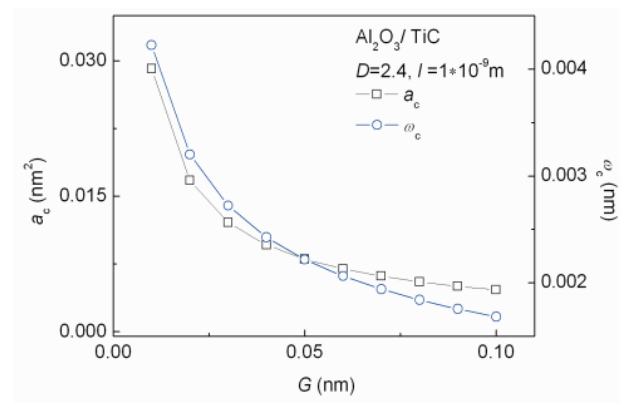

(b) $a_{c} \& \omega_{c} v s . G$

Figure 4: Critical parameters as functions of 3-D fractal parameters for Al2O3/ TiC contacting system listed in Table 1.

Figs. 3 illustrates the effect of the asperity's base diameter on the critical parameters, $\omega_{\mathrm{c}}, a_{\mathrm{c}}$ and $\Delta F_{\mathrm{e}}\left(a_{\mathrm{c}}\right)$ of 3-D fractal contact surfaces from three different contact systems listed in Table 1 guided from Ref. [11], from which we can see that critical parameters all tend to increase gradually with the asperity's base size and the increasing amplitude of surfaces with different material properties varies. 
Figs. 4 displays that the critical parameters $a_{\mathrm{c}}$ and $\omega_{\mathrm{c}}$ vary with the fractal parameters $D$ and $G$ of 3-D fractal surfaces in $\mathrm{Al}_{2} \mathrm{O}_{3} / \mathrm{TiC}$ contacting system, from which it can be seen the increase and decrease in $a_{\mathrm{c}}$ and $\omega_{\mathrm{c}}$ with increasing $D$ and $G$ respectively. The results of the above figures reveal strong dependence of critical parameters, $\omega_{\mathrm{c}}, a_{\mathrm{c}}$ on fractal parameters, the asperity's base diameter and material properties, i.e. they are fractal but scale-dependent.

The analysis in last section also suggests that the relationship between the contact area and the contact load of an elastically deformed fractal asperity satisfies $a \propto \Delta F_{\mathrm{e}}{ }^{2 / 3}$ as shown in Eqs. 18 and 22, in agreement with classical contact mechanics. However, load-area relationships given by Refs. [3] and [7] for 2-D and 3-D fractal surfaces respectively are shown in Eq. 25.

$$
\Delta F_{\mathrm{e}}=\left(4 \pi^{1 / 2} E G^{(D-1)} / 3\right) a^{(3-D) / 2}, 1<D<2 \text {, and } \Delta F_{\mathrm{e}}=\left[2^{(15-3 D) / 2}(\ln \gamma)^{1 / 2} E G^{(D-2)} / 3 \pi^{(4-D) / 2}\right] a^{(4-D) / 2}, 2<D<3
$$

It is clear to see that the above area-load relationships all vary from $a \propto P$ to $a \propto P^{2}$. This difference between the presented revised fractal model and the existing fractal models is also due to the assumption that $a$ always equals $l^{2}$ and $\omega \equiv \delta$.

In addition, although the transformation rule for a fractal asperity transfers from an elastic contact to a plastic contact and the power-law behavior between the contact load and the contact area obtained in this study is similar to that in Ref. [13], the equations derivation process in this study is more simple and acceptable. The critical deformation $\omega_{\mathrm{c}}(0 \leq \omega \leq \delta)$ obtained just by setting equal to the asperity's critical height $\delta_{\mathrm{c}}$ in Ref. [13] is arbitrary and not acceptable. However, the expression of $\omega_{\mathrm{c}}$ in this study was deduced strictly and convincingly in an easier way. Moreover, in this paper, the analysis was expanded to the contact mechanics of 3-D fractal rough surfaces; also the numerical study was conducted to investigate the effect of material properties, the size of asperity's base and fractal parameters on the critical parameters.

\section{Conclusions}

In this paper, the unconvincing aspects in Ref. [13] was resolved and the existing elastic-plastic contact models of a single asperity with fractal geometry were revised to investigate the scale-dependence of the critical parameters and relationships between the contact area and contact load. Numerical analysis was conducted to further study the influences of the asperity's base diameter, material properties and fractal parameters on the critical parameters. Based on the presented analysis and discussion, the following main conclusions can be drawn from this study.

(1) The asperity's critical deformation $\omega_{\mathrm{c}}$ obtained in this study is objective and reasonable, and the analysis process is clearer and simpler.

(2) Critical parameters, including critical deformation, critical contact area and critical contact load are all scale-dependent on the asperity's base size, and determined jointly by the asperity's base size, fractal parameters and material properties.

(3) The condition for a fractal asperity transfers from elastic contact to plastic contact is consistent with that in classical contact mechanics but contrary to that in the M-B model.

(4) The relationship between the contact load and the contact area of an elastically deformed fractal asperity satisfies $a \propto \Delta F_{\mathrm{e}}^{2 / 3}$ as in agreement with classical contact mechanics but in disagreement with the existing fractal contact models.

\section{Acknowledgements}

This work was financially supported by the National Natural Science Foundation of China (51505126), the Fundamental Research Funds for the Central Universities (BZX/13B101-02) and Applied Basic Research Programs of Science and Technology Commission Foundation of Jiangsu Province (BK20150256). 


\section{References}

[1] J. Greenwood, J. Tripp, The contact of two nominally flat rough surfaces, P. I. Mech. Eng. 185(1970) 625-634.

[2] P. R. Nayak, Random process model of rough surfaces in plastic contact, Wear. 26 (1973) 305-333.

[3] A. Majumdar, B. Bhushan, Fractal model of elastic-plastic contact between rough surfaces, J. Tribol-T. Asme. 113 (1991) 1-11.

[4] S. Wang, K. Komvopoulos, A fractal theory of the interfacial temperature distribution in the slow sliding regime: Part I Elastic contact and heat transfer analysis, J. Tribol. 116 (1994) 812-822.

[5] S. Wang, K. Komvopoulos, A Fractal Theory of the Interfacial Temperature Distribution in the Slow Sliding Regime: Part II Multiple Domains, Elastoplastic Contacts and Applications, J. Tribol. 116 (1994) 824-832.

[6] S. Wang, K. Komvopoulos, A fractal theory of the temperature distribution at elastic contacts of fast sliding surfaces, J. Tribol. 117 (1995) 203-214.

[7] W. Yan, K. Komvopoulos, Contact analysis of elastic-plastic fractal surfaces, J. Appl. Phys. 84(1998) 3617-3624.

[8] M. Berry, Z. Lewis, On the Weierstrass-Mandelbrot fractal function, P. Roy. Soc. A-Math. Phy. 370 (1980) 459-484.

[9] K. Komvopoulos, Z. Gong, Stress analysis of a layered elastic solid in contact with a rough surface exhibiting fractal behavior, Int. J. Solids Struct. 44 (2007) 2109-2129.

[10]K. Komvopoulos, Effects of multi-scale roughness and frictional heating on solid body contact deformation, CR Mecanique 336 (2008) 149-162.

[11]X. Yin, K. Komvopoulos, An adhesive wear model of fractal surfaces in normal contact, Int. J. Solids Struct. 47 (2010) 912-921.

[12]S. Jiang and Y. Zheng, An analytical model of thermal contact resistance based on the Weierstrass-Mandelbrot fractal function, P. I. Mech. Eng. C-J Mec. 224 (2010) 959-967.

[13] Y. Morag, I. Etsion, Resolving the contradiction of asperities plastic to elastic mode transition in current contact models of fractal rough surfaces, Wear 262 (2007) 624-629. 